DOI: https://doi.org/10.46296/ig.v2i4.0010

\title{
PRODUCCIÓN DE LICOR DE PÉTALOS DE ROSAS EN RÉGIMEN SEMICONTINUO
}

\section{PRODUCTION OF ROSE PETALS LIQUOR IN A SEMICONTINUOUS REGIME}

\author{
Mendoza-Pico Vicky ${ }^{1 *}$ García-Mujica Robert ${ }^{2}$; Morales-Paredes Carlos Augusto ${ }^{3}$ \\ ${ }^{1}$ Universidad de Jaén, UJA. Jaén, España. \\ ${ }^{2}$ Universidad Técnica de Manabí, UTM. Portoviejo, Ecuador. \\ ${ }^{3}$ Universidad Laica Eloy Alfaro de Manabí, ULEAM. Manta, Ecuador.
}

*Correo: vmendoza8924@gmail.com

\begin{abstract}
Resumen
El presente manuscrito se basa en el estudio del escalamiento de la producción de un licor a base de pétalos de rosas, donde se establecen los principales parámetros de investigación que involucran llevar a cabo un análisis de la materia prima y la relación entre materia prima y producto final. A continuación, se diseñó el proceso productivo a escala laboratorio como punto de partida para obtener un balance de materiales y poder pasar de escala de laboratorio a una producción en régimen semicontinuo para una producción final de $50 \mathrm{~L}$ por lote. Con los datos de la experimentación y el escalado se realizó un plano de la distribución del proceso productivo en la planta, el cual se desarrolla en 7 etapas; se destaca como proceso principal, la técnica de extracción solido-líquido que es donde interviene la extracción del aceite esencial de los pétalos de rosas por medio de la utilización de un solvente que para este caso es el etanol a $95^{\circ}$. También se definieron los equipos de la planta necesarios para cumplir con el volumen de producción final. El proyecto finaliza con el análisis físicoquímico del producto, donde se estableció que el licor cumple con las Normas INEN 1837 para la elaboración de bebidas alcohólicas en específico para licores, obteniendo en este producto un grado alcohólico de $25^{\circ}$. Finalmente, se analizó la presencia de metanol y furfural que dieron negativos para el producto.
\end{abstract}

Palabras clave: licor, aceite esencial, rosas, licor de rosas, semicontinuo.

\begin{abstract}
This article is based on the study of the scaling of the production of a liquor based on rose petals, where the main research parameters that involve carrying out an analysis of the raw material and the relationship between raw material and product are established. final. Next, the laboratory scale production process was designed as a starting point to obtain a balance of materials and to be able to go from laboratory scale to industrial scale production for a final production of $50 \mathrm{~L}$ per batch. With the data from the experimentation and the scaling, a plan of the distribution of the production process in the plant was carried out, which is developed in 7 processes, of which the solid-liquid extraction stands out as the main process, which is where the extraction of the essential oil of rose petals by using the solvent that in this case is ethanol at $95^{\circ}$. The plant equipment necessary to meet the final production volume was also defined. The project ends with the physical-chemical analysis of the final product, where it was established that the liquor complies with the INEN 1837 Standards for the manufacture of alcoholic beverages specifically for liqueurs, obtaining in our product an alcoholic degree of $25^{\circ}$, the presence of methanol and furfural that were negative for the product.
\end{abstract}

Keywords: liquor, essential oil, roses, rose liquor, semi-continuous.

Información del manuscrito:

Fecha de recepción: 10 de mayo de 2019

Fecha de aceptación: 27 de junio de 2019

Fecha de publicación: 10 de julio de 2019 


\section{Introducción}

En Ecuador, la floricultura está considerada como uno de los sectores agrícolas de mayor relevancia en la economía del país. Se considera como la tercera actividad agrícola que mayores réditos económicos aporta al país (Cerón, 2017).

Según las estadísticas de la Corporación Financiera Nacional (CFN, 2016), se llegaron a producir casi 6 billones de tallos en al año 2016, de los cuales el $68 \%$ correspondía a la cosecha de rosas. El uso principal de estas flores ha sido como elementos ornamentales, su aceite esencial en especial se ha utilizado para la elaboración de perfumes y jabones.

En Ecuador se cultivan diferentes variedades de rosas, convirtiéndonos en un país con gran número de hectáreas cultivadas y produciendo la gama más variada de colores (Cabrera, 2011). Por tanto, el objetivo del presente trabajo es proponer una alternativa para las rosas cultivadas en el Ecuador y que además por la alta producción de estas se puede aprovechar este recurso, así como los residuos y desechos que generan las rosas. Se propone la elaboración de bebidas alcohólicas como una alternativa innovadora en el medio. Para elaborar bebidas alcohólicas a lo largo del tiempo se han utilizado variedades de materias primas que van desde la cebada hasta el maíz, utilizando también el arroz o una mezcla de sustratos (Márquez, 2015).

Desde otra perspectiva, los licores que contemplan flores como: rosa o lavanda como ingrediente principal resultan muy apreciados por el característico gusto que imprimen en el paladar, durante el renacimiento del licor obtenido por maceración de pétalos de rosa con miel, conocido como rosoli fue muy popular en la corte florentina, en la actualidad se produce artesanalmente un licor de rosas en combinación con otras especies vegetales en la isla de Menorca (Valencia, 2012).

Las flores desde siempre han formado parte de nuestra dieta, estas contienen grandes cantidades de nutrientes (Druitt, 2004). Los pétalos de rosa siendo parte de los ingredientes de una receta de cocina no es algo nuevo, esto gracias a su agradable sabor, color y aroma, lo que hace más atractivos distintos 
platos, vinos y licores (Anchundia, 1999; Park et al., 2016).

Actualmente no existen empresas productoras de licores de pétalos de rosas en la provincia de Manabí en una condición formal según datos del INEN y los licores existentes son el resultado de importaciones, existen lugares donde se elabora licor de forma artesanal como cocteles, cuya producción no es significativa, ya que sus productos se consumen en el mismo local o bar (Lastra, 2012).

En este proyecto se llevó acabo el estudio y desarrollo de un licor a base de pétalos de rosas, de modo que también se planteó el diseño de la planta. Es así como los resultados de este estudio establecen los equipamientos y requisitos necesarios para llevar acabo todo el proceso de producción optimizando los recursos necesarios.

Para obtener el producto principal denominado como licor de rosas se utiliza la extracción sólido-líquido como punto de partida en la elaboración del licor, esta técnica se complementa con el proceso de mezclado donde se obtiene un producto final de calidad que se ajusta las normas establecidas.

\section{Metodología}

\subsection{Descripción del proceso}

Para llevar a cabo la elaboración del licor a base de pétalos de rosas se utilizó una metodología experimental, teniendo como punto de partida un ensayo de laboratorio que posteriormente se transformó en una producción en régimen semicontinuo. Esta investigación va de la mano con un conjunto de fundamentos bibliográficos basados en los distintos procesos para elaborar el licor a base de pétalos de rosas y otros subproductos.

En la literatura revisada se denota que la técnica de maceración es comúnmente empleada para convertir el aceite esencial de los pétalos de rosas en el licor, utilizando etanol como solvente de la maceración. Otro método encontrado en bibliografía es la destilación por arrastre de vapor y posterior separación del agua y el aceite esencial, que una vez obtenido pasa a un mezclado con etanol y dilución.

Se midieron los grados Gay Lussac del etanol como parámetro de control de calidad de los licores, para lo cual 
se utilizó un alcoholímetro con una escala de 0-100 G.L.

Para la producción específica del licor a base de pétalos de rosas se utilizó como técnica principal la extracción sólido-líquido, también conocida como extracción Soxhlet, que se utiliza principalmente para obtener el aceite esencial de los compuestos, en este caso específico se utilizó para obtener el aceite esencial de los pétalos de las rosas, utilizando los cartuchos del equipo diseñados para cargar la muestra y circular sobre la misma el solvente, que para esta investigación fue etanol a 95 G.L.

Para llevar a cabo el escalamiento al régimen semicontinuo se partió de los datos obtenidos experimentalmente mediante un balance de materia, el cual permitió estimar la cantidad de producto final $y$ de desechos que se generan en el proceso, para así definir todas las etapas del proceso de producción.

Se definieron los equipos a utilizar por medio de un balance de materia en régimen semicontinuo y un diagrama de procesos, de donde se partió para establecer la capacidad de producción de cada equipo y el costo del mismo.
Por último, se llevaron a cabo los análisis físicos-químicos del producto final, mediante una cata se obtuvieron el color, sabor y olor del licor de rosas. Además, se realizaron pruebas de presencia de metanol y furfural para descartar contaminantes 0 productos indeseados en el licor.

\subsection{Proceso experimental}

Desde el punto de vista experimental, se utilizó como punto de referencia el estudio realizado por Valencia (2012), quien menciona que para la preparación de licor a base de pétalos de rosa se aplica un proceso de extracción utilizando vapor de agua para obtener el aceite esencial y mezclarlo con alcohol, lo que conlleva posteriormente a una extracción sólido-líquido en un lecho estático. El presente estudio se sustenta en un experimento que emplea la extracción sólido-líquido mediante la utilización del equipo Soxhlet, donde el cartucho se carga con los pétalos de rosas y se utiliza directamente el etanol $\left(96^{\circ}\right)$ como solvente, esta técnica permite simplificar los procesos y reducir tiempo de producción, debido a que el solvente no es recuperado por ser parte del producto final. Luego el 
alcohol es mezclado con agua mineral lo que permite reducir la concentración de grados Brix, según las especificaciones de la norma INEN reguladoras del consumo humano de licor.

\section{Resultados y discusión}

\subsection{Demanda}

Ecuador, con 3.000 hectáreas, es el principal productor de rosas en extensión, superando a Colombia, Kenia y Etiopía, y gracias a las condiciones climáticas, produce más de 400 variedades con características únicas, como tallos de unos 2,20 metros de altura (Cabrera, 2011).

Desde el punto de vista del licor de rosas su clasificación con relación a la demanda estaría situada en bienes no necesarios o de gusto, lo que se conoce comúnmente como consumo suntuario, dentro de esta categoría se encuentran también ropa fina, perfumes y otros bienes.

En este punto el consumidor realiza una compra con la intención de satisfacer un gusto y no una necesidad. Es decir que el escenario de la compra del licor de rosas dependerá mucho de los gustos del cliente.
Los consumidores presentan características como las siguientes:

- Una gran mayoría de la población no sabe de la existencia de un licor con olor a rosas y de los beneficios que pueden adquirir al consumirlo de forma moderada.

- Como en el mercado se presentan licores importados que se establecen bajo un alto precio, los clientes tienen la idea de que todos los licores se rigen bajo el mismo valor.

- Consideran que el licor de rosas posee el mismo sabor que los licores normales con la característica de su olor.

El licor de rosas está dirigido a todas las personas entre 18 y 65 años, sin importar su ocupación, raza, religión, cultura o género, que deseen probar una nueva bebida de moderación de buen sabor y a un costo conveniente.

\subsection{Balance de materiales a escala de laboratorio}

El balance de materiales para la producción de un licor a base de pétalos de rosa se explica en la figura 1.

Como se observa en la figura 1 , se inicia con 14 gramos de pétalos de rosas cortados que ingresan al 
cartucho del equipo Soxhlet y 200 $\mathrm{mL}$ de etanol a 95 G.L., este último actúa como solvente para llevar a cabo la extracción del aceite esencial. Al finalizar el primer proceso se obtienen $145 \mathrm{~mL}$ de licor de rosas a 93 G.L. que son llevados a la segunda fase donde son necesarios $500 \mathrm{~mL}$ de agua desmineralizada para obtener un total de $645 \mathrm{~mL}$ a 25 G.L., que es lo que establece la norma INEN 1837 en sus parámetros para que un licor pueda ser consumido.

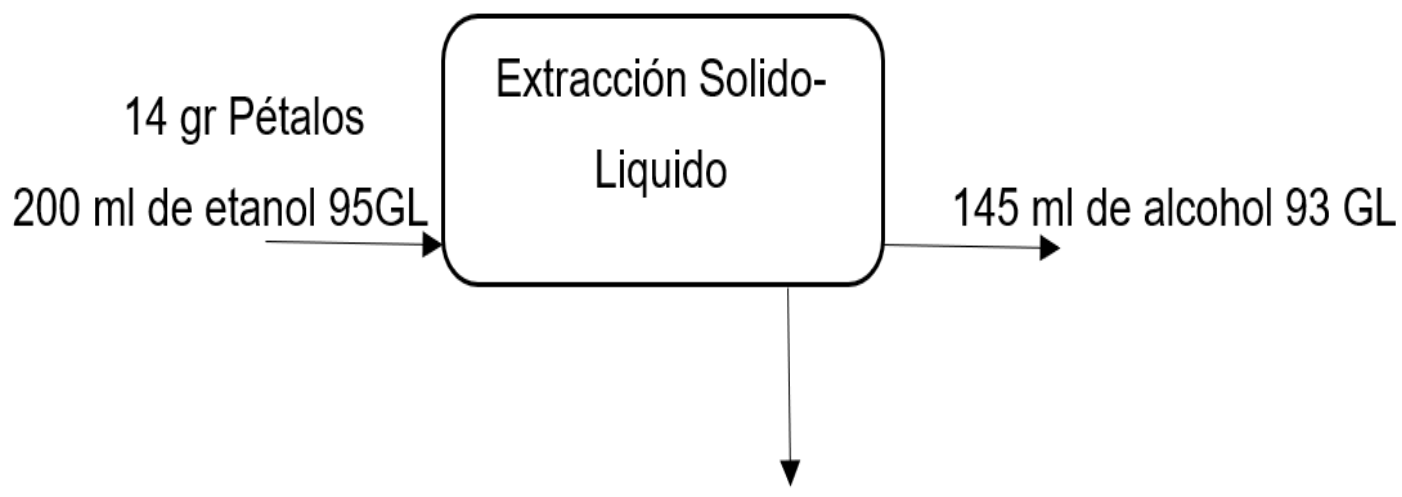

Residuos

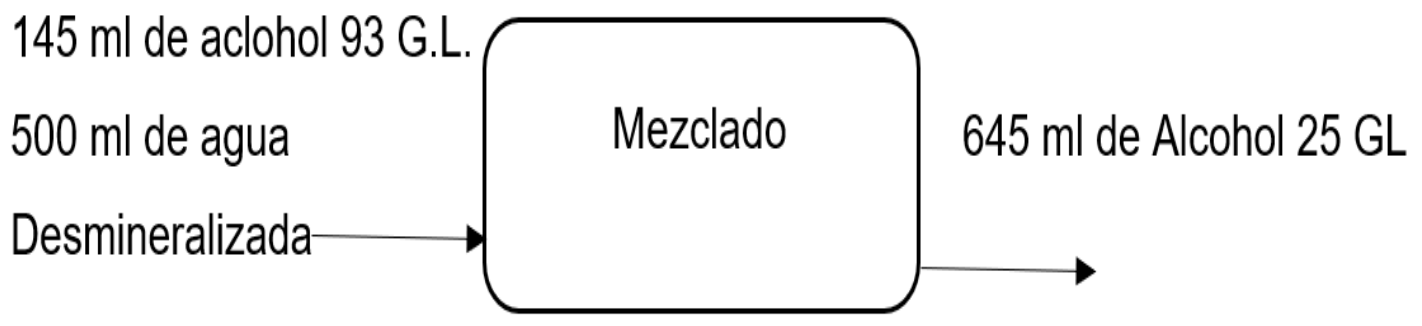

Figura 1. Balance de masa a escala batch

Según la bibliografía reportada, 500 gramos de pétalos de rosas rojas orgánicas se mezclan en un litro de etanol y se colocan en maceración durante 3 semanas, pasado este tiempo se obtuvieron 0,95 litros de Alcohol al 95 G.L. y quedaron los residuos de los pétalos de rosas. Después de esto el etanol obtenido pasa a ser rebajado con agua desmineralizada, requiriéndose un volumen de 4 litros para reducir la concentración hasta 25 G.L. (Valencia, 2012). Sin embargo, el 
proceso de maceración por si solo puede llevar consigo el arrastre de componentes de las rosas que no son orgánicos y que pueden presentarse como sustancias contaminantes, pesticidas 0 germicidas, 0 en su defecto la maceración puede generar la formación de metanol en el producto final.

\subsection{Determinación del solvente extraído}

Los datos que se obtuvieron experimentalmente son los siguientes: el total de materia prima para extraer es 14 gramos y el solvente a utilizar es etanol con un volumen de $250 \mathrm{~mL}$ y una densidad de $789 \mathrm{Kg} / \mathrm{m}^{3}$; la cantidad de etanol que se obtuvo al finalizar la extracción fue $150 \mathrm{~mL}$, además la bibliografía señala que el $1 \%$ en peso de pétalos de rosa corresponde al contenido de aceite esencial; con estos valores se puede llevar a cabo el cálculo del solvente total extraído, tal y como se muestra en la figura 2 .

$$
\begin{array}{ll}
V_{1}=? & V_{2}=250 \mathrm{ml}=197,25 \mathrm{gr} \\
X_{A 1}=? & X_{A 2}=?
\end{array}
$$

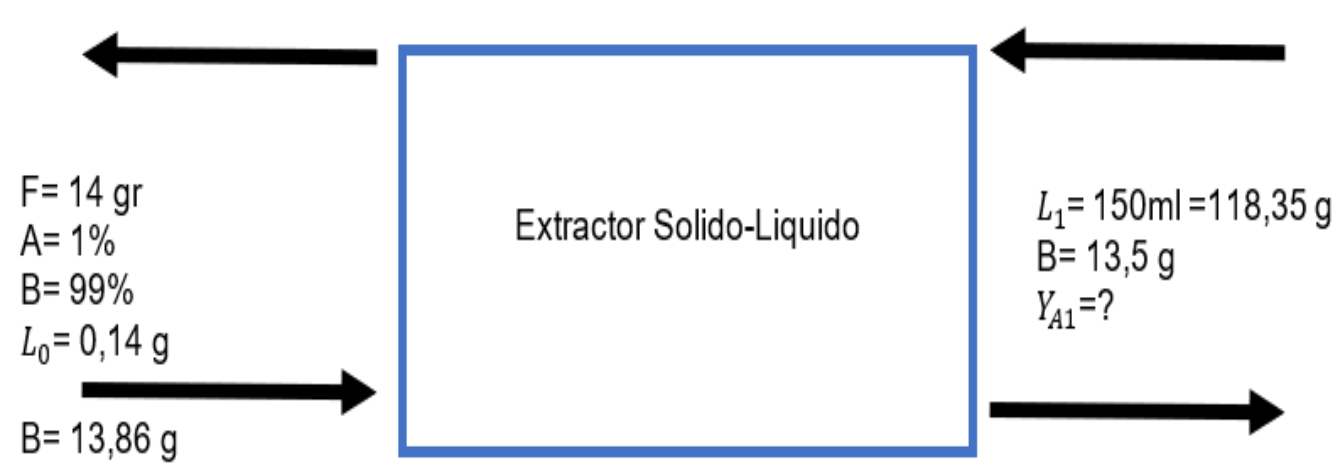

Figura 2. Diagrama para la determinación del aceite esencial extraído

Del diagrama establecido en la figura 2 se utilizan los cálculos de extracción sólido-líquido que permitieron determinar que la cantidad de aceite esencial extraído fue 0,0894 gramos y 118.35 gramos de solución final. 


\subsection{Escalado a régimen semicontinuo}

Inicialmente la capacidad de trabajo de la planta está concebida para procesar $1 \mathrm{Kg}$ de pétalos de rosas y para llevar a cabo su proceso productivo es necesario utilizar $20 \mathrm{~L}$ de etanol a 95 G.L. como solvente en la etapa de extracción sólido-líquido. Posteriormente, para pasar a la etapa de mezclado se deben agregar $11,22 \mathrm{~mL}$ de alcohol al 93 G.L. que junto con $39 \mathrm{~L}$ de agua desmineralizada van a dar como producto final $50 \mathrm{~L}$ de licor de rosas listos para su distribución y consumo (Figura 3).
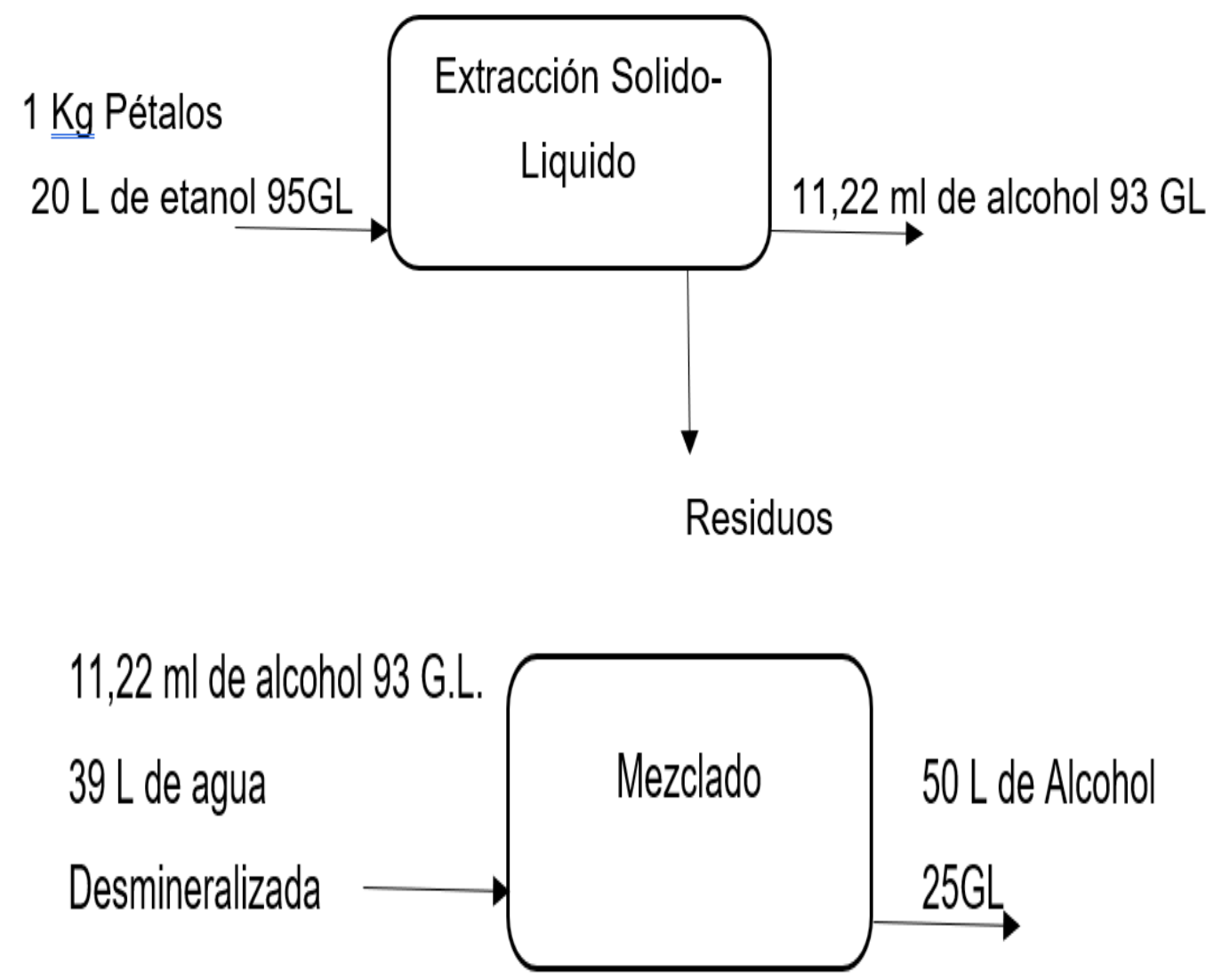

Figura 3. Balance de materiales para una producción a escala industrial

Industrialmente, una planta para la producción de licor a base de pétalos de rosa debe contar con el equipamiento necesario que permita desarrollar todo el proceso productivo. Es por este motivo que se definen las siguientes operaciones unitarias (figura 4). 


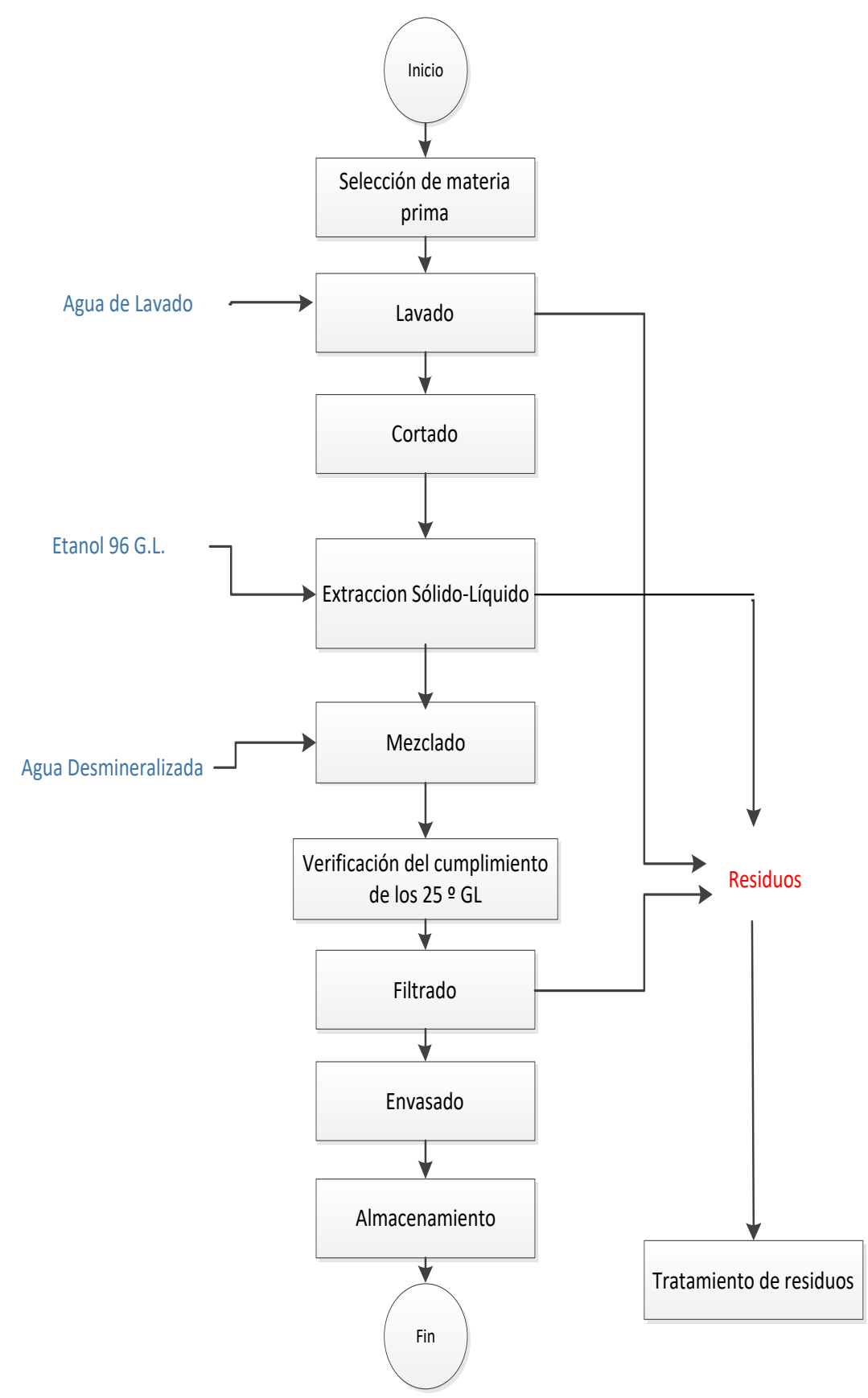

Figura 4. Diagrama de bloques del proceso de producción de licor de rosas

La primera etapa corresponde a la recepción y selección de la materia prima (pétalos de rosas), seguido de la etapa de lavado y cortado de los pétalos, operación que se lleva a cabo manualmente, la tercera fase es el proceso más importante, puesto que se genera la extracción sólido-líquido utilizando como solvente el etanol a 96 G.L., una vez 
terminada la extracción del aceite esencial se pasa a la cuarta fase, el mezclado, donde se utiliza el agua desmineralizada para diluir la solución alcohólica, desde una concentración de 93 G.L. a 25 G.L., correspondiente a lo que se establece en la norma INEN1837, el producto pasa a la etapa de filtrado para eliminar partículas suspendidas $y$ finalmente el producto se encuentra listo para ser envasado y almacenado.

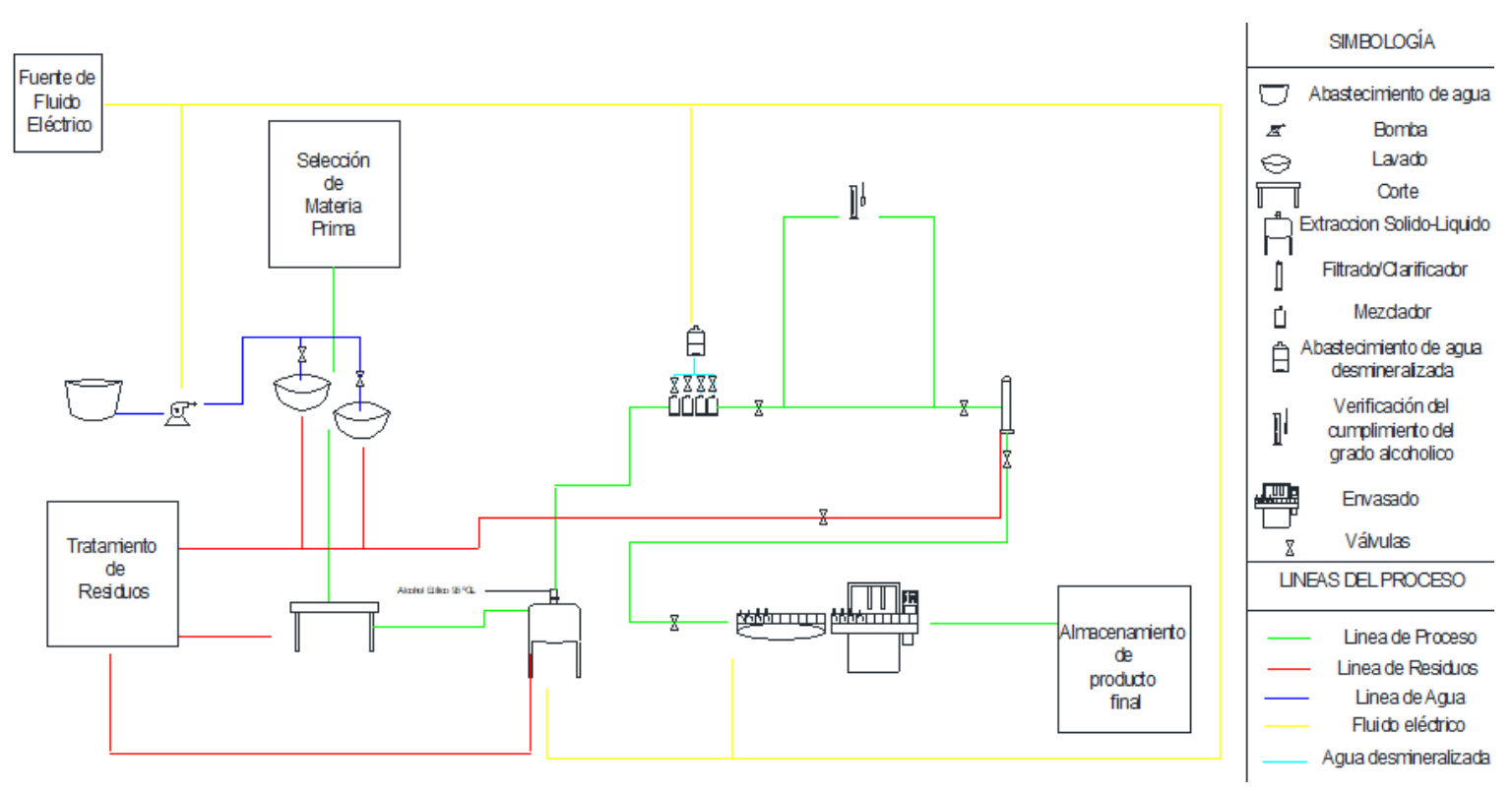

Figura 5. Diagrama de procesos para la producción de licor de rosas

En la figura 5 se observa una adecuada distribución de la zona de producción de la planta de licor de rosas, tomando en cuenta que las líneas de proceso son unidireccionales, se muestra también el flujo de las líneas de electricidad y de agua que son necesarias para el correcto funcionamiento de la planta. El precio del litro de licor establecido por el Servicio de Rentas Internas de es $\$ 7,25$ el litro, usando un valor promedio de $\$ 8,00$ el litro.

\subsection{Equipos}

Para un funcionamiento optimizado de la planta de licor de rosas se seleccionaron los equipos más adecuados, es decir que su capacidad está adaptada al respectivo escalamiento del proceso, además se establece el equipo que de una mayor funcionabilidad durante proceso 
productivo. Los equipos y sus costos se describen en la tabla 1.

Tabla 1.

Equipamiento necesario para implementar el proceso a escala industrial

\begin{tabular}{|c|c|c|c|}
\hline Nombre y Marca & Capacidad & Dimensiones & $\begin{array}{c}\text { Costo } \\
\text { Unitario }\end{array}$ \\
\hline $\begin{array}{l}\text { Recipiente para } \\
\text { lavado }\end{array}$ & 100 litros & $\begin{array}{l}\text { Altura: } 0.30 \mathrm{~m} \\
\text { Diámetro: } 1 \mathrm{~m}\end{array}$ & $\$ 40$ \\
\hline $\begin{array}{l}\text { Frasco de vidrio con } \\
\text { tapón de vidrio }\end{array}$ & 10 litros & $\begin{array}{l}\text { Altura con } \\
\text { tapón: } 0.41 \mathrm{~m} \\
\text { Diámetro de } \\
\text { cuerpo: } 0.23 \mathrm{~m}\end{array}$ & $\$ 180$ \\
\hline $\begin{array}{l}\text { Filtro/ Clarificador } \\
\text { 3MTM Betapure } \mathrm{TM}\end{array}$ & $961 / \mathrm{min}$ & $\begin{array}{l}\text { Diámetro de } \\
\text { cartucho: } \\
\text { Interno: } 28 \mathrm{~mm} \\
\text { Externo: } 64 \\
\text { mm } \\
\text { Altura: } 1 \mathrm{~m}\end{array}$ & $\$ 450$ \\
\hline $\begin{array}{l}\text { Maquina } \\
\text { embotelladora } \\
\text { ASTIMEC }\end{array}$ & 100 litros & $\begin{array}{l}\text { Altura: } 1 \mathrm{~m} \\
\text { Ancho: } 1.8 \mathrm{~m} \\
\text { Fondo: } 0.6 \mathrm{~m}\end{array}$ & $\$ 2400$ \\
\hline $\begin{array}{l}\text { Equipo para estudio } \\
\text { de extracción } \\
\text { sólido- líquido por } \\
\text { Soxhlet }\end{array}$ & 10 litros & $\begin{array}{l}\text { Altura: } 1,50 \mathrm{~m} \\
\text { Ancho: } 1 \mathrm{~m}\end{array}$ & $\$ 500$ \\
\hline
\end{tabular}

\subsection{Características del producto final}

Se realizaron los respectivos análisis del producto final obtenido a lo largo proceso productivo y se pudieron determinar los parámetros más destacados que están establecidos en la Norma INEN 1837.

Según la norma INEN 1837 para Bebidas Alcohólicas. Licores se especifica principalmente que:
- El agua utilizada para hidratar el producto hasta los niveles establecidos en la norma debe ser potable (según Norma INEN 1 108). También podrá ser desmineralizada, desionizada 0 destilada.

- Los licores deben cumplir con los requisitos establecidos en la tabla 2.

- Los licores pueden adicionarse con sustancias aromáticas y/o edulcorantes de uso permitido.

Tabla 2.

Norma INEN para Licores

\begin{tabular}{|l|c|c|c|}
\hline \multicolumn{1}{|c|}{ REQU ISITOS } & \multirow{2}{*}{ UNIDAD } & \multicolumn{2}{c|}{ A } \\
\cline { 2 - 4 } & & Mín. & Máx. \\
\hline Grado alcohólico a $15^{\circ} \mathrm{C}$ & GL & 15 & 45 \\
Acidez total, como ácido acético * & - & 1,5 \\
Esteres, como acetato de & & & \\
etilo & $*$ & - & 2,0 \\
Aldehídos, como etanal & $*$ & - & 0,5 \\
Furfural & $*$ & - & 0 \\
Alcoholes superiores & $*$ & - & 0,5 \\
Metanol & $*$ & - & 2 \\
\hline
\end{tabular}

Dentro de los análisis realizados se obtuvieron parámetros sensoriales, como olor, color y sabor. Por medio de una catación se logró valorar los parámetros organolépticos, obteniendo los siguientes resultados: el color es Rosa; el Olor es de Rosas; y el sabor de Licor (Aguardiente) (Scalliet et al., 2006). 
También se realizaron pruebas para determinar presencia de ciertos compuestos, como furfural $y$ metanol, donde se determinó que no existe la presencia de estos compuestos, ya que el método de obtención del aceite esencial es por evaporación-condensación del solvente, que es el etanol, por lo que esto no generaría una descompensación de las moléculas y no existirían compuestos indeseados (Zvi et al., 2012). Además, se verificó el cumplimiento de la concentración de alcohol (G.L.) y se estableció como base que el producto posee 25 G.L., por lo anteriormente mencionado se define que el producto de licor de rosas cumple con lo establecido en la legislación ecuatoriana.

Cabe recalcar que $1 \mathrm{~kg}$ de pétalos de rosas en conjunto con $20 \mathrm{~L}$ de alcohol y $36 \quad L$ de agua desmineralizada dan como resultado $50 \mathrm{~L}$ de licor de rosas que potencialmente se pueden vender a $\$ 8,00$ / litro; por tanto, se obtiene un valor aproximado de $\$ 12.000$ al mes, al año representa $\$ 144.000$ tomando en cuenta 360 días del año, lo que representaría en porcentaje $0,64 \%$ del mercado al que se llegaría de acuerdo con el porcentaje anual dado por el Banco Central del Ecuador.

\section{Conclusiones}

Los pétalos de rosas poseen una característica en particular que es representada por su olor, esto se debe al compuesto 2-feniletanol que puede ser aprovechado en distintos usos a lo largo de la vida cotidiana, y entre esos extensos usos se encuentra la elaboración de un licor a base del aceite esencial presente en los pétalos de rosas.

Para el proceso productivo de este licor se estableció como una operación unitaria fundamental la extracción sólido-líquido que se realiza utilizando como solvente el etanol a 95 G.L. y se definen procesos sucesivos como el mezclado y filtrado para asegurar la calidad e inocuidad del producto.

Experimentalmente se obtiene de la extracción sólido-líquido $\quad 0,08$ gramos de aceite esencial de los 0,14 gramos iniciales que ingresan a la extracción. De la misma se obtienen $150 \mathrm{~mL}$ de solución, que contiene el solvente y el soluto. El solvente no es recuperable por ser parte del producto final en cuestión. 


\section{Bibliografía}

Anchundia, P. (1999). Obtención de Aceite Esencial a Partir de Pétalos de Rosas. Trabajo de Titulación de Grado. Ingeniero Químico. Universidad Central del Ecuador. Escuela de Ingeniería Química. Quito.

Cabrera, M. (2011). Producción, Comercialización,

Exportaciones de las Flores en el Ecuador y su rol en las Exportaciones no Tradicionales (2007-2010).

Cerón, S. (2017). Propuesta de norma técnica de calidad para la producción y exportación de rosas a los principales socios comerciales de Ecuador en la Unión Europea (Alemania, España, Italia, Holanda). (Tesis de maestría, Pontificia Universidad Católica del Ecuador).

CFN. (2016). Ficha Sectorial: Cultivo de flores. Corporación Financiera Nacional. Disponible en: https://www.cfn.fin.ec/wpcontent/uploads/2017/10/FSCultivo-de-Flores-octubre2017.pdf

Druitt, L. (2004). The organic rose garden. Taylor Trade Publishing.

INEN. (2016). NTE INEN 1837. Bebidas alcohólicas. Requisitos. Disponible en: https://181.112.149.204/buzo n/normas/nte_inen_1837-

2.pdf

Lastra, J. L. (2012). Plan para la Elaboración de Licores de Frutas Tropicales del Ecuador. Trabajo De Titulación Previo a obtener el Título de Diplomado Superior en Gestión de Proyectos, Escuela Politécnica del Ejército, Sangolquí, Ecuador.

Márquez-Farías, A. J. (2015). Elaboración de una cerveza orgánica a partir de la quinoa (chenopodium quinoa) (Bachelor's thesis, Machala: Universidad Técnica de Machala).

Park, D., Shin, K., Choi, Y., Guo, H., Cha, Y., Kim, S. H., ... \& Choi, E. K. (2016). Antimicrobial activities of ethanol and butanol fractions of white rose petal extract. Regulatory Toxicology and Pharmacology, 76, 57-62.

Scalliet, G., Lionnet, C., Le Bechec, M., Dutron, L., Magnard, J. L., Baudino, S., ... \& Dumas, C. (2006). Role of petal-specific orcinol O-methyltransferases in the evolution of rose scent. Plant physiology, 140(1), 1829.

Valencia-Villacis, M. F. (2012). Aprovechamiento de pétalos de rosa comestible para la producción de licor.

Zvi, M. M. B., Shklarman, E., Masci, T., Kalev, H., Debener, T., Shafir, S., ... \& Vainstein, A. 
(2012). PAP1 transcription factor enhances production of phenylpropanoid and terpenoid scent compounds in rose flowers. New Phytologist, 195(2), 335-345. 